\title{
Digital shinrin-yoku: do nature experiences in virtual reality reduce stress and increase well-being as strongly as similar experiences in a physical forest?
}

\author{
Gerhard Reese $^{1}$ D $\cdot$ Jasmin Stahlberg $^{1} \cdot$ Claudia Menzel $^{1}(\mathbb{D}$
}

Received: 9 July 2021 / Accepted: 14 January 2022 / Published online: 3 February 2022

(c) The Author(s) 2022

\begin{abstract}
Shinrin-yoku or forest bathing refers to a therapeutic, immersive nature experience that aids to improve well-being. The goal of the current research was to compare the effects of a physical urban nature versus virtual nature experience on stress, affect, vitality, and restoration. Previous research suggested that an immersive nature experience—such as shinrin-yoku—can be beneficial for health, but direct comparisons between physical and virtual reality (VR) experiences are scarce. In the current study, fifty participants navigated self-paced through a forest scene that was either an urban physical forest or an immersive VR forest with similar characteristics as the physical one. Before and after the intervention, we measured positive and negative affect, subjective vitality, and perceived daily stress. After the intervention, we measured perceived restorative outcomes. Results revealed that both VR and physical nature experience resulted in expected effects on well-being indicators: Affect was more positive and less negative, subjective vitality increased slightly, and stress decreased slightly after both interventions. There were no significant differences between the two settings on any of the variables, but slightly stronger effect sizes over time within the physical condition. Overall, these findings suggest that immersive VR nature experiences can have restoration effects similar to physical nature experiences, suggesting intervention strategies when physical nature options are scarce.
\end{abstract}

Keywords Virtual reality $\cdot$ Restoration $\cdot$ Stress $\cdot$ Shinrin-yoku $\cdot$ Urban forest

\section{Introduction}

Natural environments are important for humans, providing food, shelter, and even symbolic or cultural meaning (e.g. Scannell and Gifford 2010). Moreover, natural environments have physiological and psychological benefits both for individuals (e.g. Berman et al. 2008; Bratman et al. 2019; Engemann et al. 2019; Hartig et al. 2014; Kuo 2015) and societies as a whole (e.g. Becker et al. 2019; Kuo and Sullivan 2001; Zelenski et al. 2015). With more and more people living in cities, urban forests and landscapes become particularly important areas for restoration. In Japan, for example, people engage in forest bathing - shinrin-yoku-in order to improve individual's health (Hansen et al. 2017). Hansen

Gerhard Reese

reese@uni-landau.de

1 Department of Social, Environmental, and Economic Psychology, Institute of Psychology, University of KoblenzLandau, Campus Landau, Fortstr. 7, 76829 Landau, Germany and colleagues' review of 64 studies suggested that practicing shinrin-yoku by "immersing oneself in nature by mindfully using all five senses" (ibid. p.1) is associated with various therapeutic effects (e.g. in terms of reducing depression, anxiety, heart rate). At the same time, more and more people in industrialized societies spend their time in environments that lack of natural elements, such as cities and buildings. In addition, as Mattila and colleagues argued (2020), based on Ilies and colleagues (Ilies et al. 2007) and De Lange and colleagues (De Lange et al. 2003), many jobs and daily hassles require constant attention and high workload, resulting in stress and long-term deterioration of well-being. Stressful work environments and commuting may also hinder physical nature experiences, thus requiring alternatives for psychological and physiological restoration. Similarly, many people may not have the physical constitution or mobility to access nature areas. It is therefore useful to assess the effectiveness of interventions that simulate natural environments through virtual reality (VR) technology. The goal of the current study was thus to validate whether a simulated forest environment through VR has comparable benefits with 
regard to indicators of well-being than a comparable urban forest environment.

\subsection{Effects of nature experiences on well-being}

Health and well-being are important aspects for a fulfilled life. According to the World Health Organization (WHO 2019), health is 'a state of complete physical, mental, and social well-being and not merely the absence of disease or infirmity' (see the first principle of the Preamble to the WHO Constitution: https://www.who.int/about/who-we-are/ constitution). Mental well-being, then, can be seen as one part of health, and can be defined as a multicomponent concept that includes affective and cognitive elements (Bratman et al. 2019). These components refer to cognitive functioning in the sense of attention and memory capacity (which are relevant within restoration theories; see below), as well as to (the lack of) mental distress and negative affect. The latter concepts in particular are often used in studies investigating effects of nature experiences on mental health.

As indicated above, immersive natural environmentsespecially when compared to urban or built environmentsare associated with several beneficial health effects (e.g. Bratman et al. 2019; Hartig et al. 2014; Kuo 2015). These effects are often discussed in relation to two influential theories in the field, namely the attention restoration theory (ART; Kaplan 1995) and stress recovery theory (SRT; Ulrich et al. 1991). These theories have been used to seek explanations for the therapeutic effects of shinrin-yoku (Hansen et al. 2017). The ART claims that voluntary attention (i.e. directed attention) depletes in urban environments and cognitively demanding tasks, and restores in natural environments (Kaplan 1995). The restorativeness of a natural environment is assumed to be based on feelings of fascination, being-away, coherence, and compatibility (Kaplan 1995). SRT in turn proposes that natural environments influence affective states and therefore facilitate recovering from stressors (Ulrich et al. 1991). For both theories, there is evidence, both in real-life settings as well as in virtual nature settings (through VR, videos, or pictures; e.g. Brown et al. 2013; Gladwell et al. 2012; Valtchanov and Ellard 2010; for meta-analyses on various aspects of restoration, see Menardo et al. 2019; Ohly et al. 2016; Stevenson et al. 2018).

Although physical urban nature experiences in particular benefit not only from natural views and sounds, but also natural odours, reduced noise, a more beneficial air composition, and a natural terrain underfoot (Alvarsson et al. 2010; Franco et al. 2017; Kuo 2015), virtual representations of nature can evoke some of the beneficial effects as well. Merely watching videos or images depicting natural environments (often compared to built environments) brings benefits in terms of current affective states, working memory, and subjective restoration and stress (Berman et al. 2008;
Berto 2005; Gamble et al. 2014; Hartig et al. 1996; van den Berg et al. 2015). With an increasing number of people having limited access to natural environments, it is important to know how comparable experiences in real and virtual natural environments are. Based on these theoretical assumptions and related findings, it is likely that an immersive, digital VR nature experiences can result in improved mental health outcomes (Mattila et al. 2020).

\subsection{Virtual reality and nature}

We focus on the possibly most elaborated digital method of presenting and experiencing visual nature input, namely VR. Previous studies suggested that immersive VR nature experiences are useful to reduce pain, stress, heart rate, and blood pressure while at the same time, they can increase restoration, vitality, and positive affect (Anderson et al. 2017; Browning et al. 2019; Gerber et al. 2017; Hedblom et al. 2019; Mattila et al. 2020; Tanja-Dijkstra et al. 2018; Yu et al. 2018; for comparisons of different presentation means, see Brivio et al. 2021). They can also benefit cancer treatment by distracting patients, reducing their frustration, increasing relaxation, and inducing a feeling of peace (Scates et al. 2020). It is thus plausible to assume that immersive VR experiences can represent a digital form of shinrin-yoku.

To our knowledge, however, there is only limited research that directly compared urban physical and VR nature experiences with regard to well-being outcomes. Chirico and Gaggioli (2019) investigated how people responded emotionally after being exposed to a real-life panoramic view of a lake or to an immersive footage of that landscape. In summary, there were no affective differences between the VR and the real condition (except for anger, which was supposedly stronger in the real nature environment, and amusement that was stronger in the VR condition). Further well-being outcomes were not measured. In a study by Mattila and colleagues (2020), participants were encouraged to look around in a VR environment for five minutes. After the intervention, participants reported stronger restoration and vitality as well as more positive affect than before. Although there was no control group, Mattila and colleagues could compare their results with data from another study assessing responses after visiting a physical forest (Hauru et al. 2012). Results of this analysis suggested that the VR environment was seen as equally restorative as a comparable physical forest, yet even more fascinating and coherent. However, this approach does not replace a proper control condition in which the procedure and content of the environment is matched.

\subsection{The present research}

The main goal of the present study is to compare the effects of VR nature on well-being-related outcomes in direct 
comparison to a walk in an urban physical forest. Specifically, participants either partook in a self-paced physical forest walk or in a self-paced VR forest walk. We decided to use a forest surrounding because previous research both in the shinrin-yoku realm but also in other contexts found evidence for their effectiveness in providing restoration (for a review, see Hansen et al. 2017; Mattila et al. 2020). Of course, there are other nature settings that can elicit restoration (such as beach surroundings; Tanja-Dijkstra et al. 2018; Reese et al. 2021). Given that physical nature may provide more factors beneficial for health (e.g. air composition, scent, soil beneath the feet etc.; for an overview, see Franco et al. 2017; Kuo 2015), it is plausible to assume that a physical nature experience is superior to a VR nature experience with regard to positive well-being outcomes. To date, however, there is insufficient research directly comparing VR and real nature environments with regard to well-being outcomes. This, however, is important in order to understand whether one or the other (or both) should be recommended in leisure and recreational but also therapeutic settings. For example, if both prove effective, health authorities could compare costs and benefits for both and provide guidance on which form of shinrin-yoku—digital or real—may be appropriate for different groups or circumstances. A recent study by Reese and colleagues (2021), for example, suggested that being guided through a VR surrounding resulted in less subjective stress than actively navigating through it. This suggests that people requiring assistance could benefit from short-term VR settings, especially when urban green is inaccessible.

Consequently, the strengths of the current study, compared to previous studies assessing the restorative effects of VR nature experiences, are that it:

(a) Provides a direct comparison between a self-paced physical nature experience and a self-paced and selfcontrolled, moving VR experience in a similar forest environment, rather than merely viewing a $360^{\circ}$ footage,

(b) Tests the effects of the different interventions on positive and negative affect, stress, vitality, subjective feelings of restoration, and perceived restorativeness of the environment in one study, and

(c) Applies a vivid VR environment resembling the physical nature environment as closely as possible.

Based on our theoretical reasoning and previous findings on the effects of VR nature experiences, we tested the following hypotheses:

(H1) For both the VR and the physical experience, people report increased positive affect, subjective vitality, as well as reduced perceived stress and negative affect after the intervention.
(H2a) We expect a stronger increase in positive affect, subjective vitality, and restoration, as well as reduced perceived stress and negative affect in the physical rather than the VR nature condition.

(H2b) Participants who completed the physical nature experience feel more subjective restoration than participants who completed the VR nature experience.

(H3) Finally, given that physical nature is likely characterized by more health-beneficial factors, we assumed that physical nature is perceived as more restorative than VR nature.

For explorative reasons, we additionally assessed how strongly participants perceived various physical characteristics of the respective environments. This analysis is not part of the main research question, and can be found in the supplemental material A.

\section{Materials and methods}

\subsection{Design and instruments}

In order to test our hypotheses, we used a 2 (Condition: VR vs. physical) $\times 2$ (Time: Before vs. after the walk) experimental design, with between variation on the first factor. We used the following measures to test our hypotheses: Positive and negative affect, perceived stress, and subjective vitality were measured before $(t 1)$ and after $(t 2)$ the intervention. Restoration outcomes and perceived restorativeness were only measured after the forest experience. We assessed demographics at the end of the second questionnaire, as well as motion sickness and previous experience with VR (in the VR condition only).

\subsubsection{Affect}

To measure affect, we used the German version of the "positive and negative affect schedule" (PANAS; Breyer and Bluemke 2016; original version: Watson et al. 1988). The scale consists of 20 items, divided into ten items measuring positive affect (e.g. "strong", "enthusiastic") and ten items measuring negative affect (e.g. "distressed", "jittery"). We asked participants how strongly they experienced the respective affect and they could respond on a 5-point-Likert-type scale from 1-not at all to 5-totally. Both scales were reliable before $(t 1)$ and after $(t 2)$ the walk, $\alpha_{t 1 \_ \text {positive }}=0.98, \alpha_{t 2 \_ \text {positive }}=0.92, \alpha_{t 1 \_ \text {negative }}=0.91$, $\alpha_{t 2 \_ \text {negative }}=0.91$. 


\subsubsection{Stress}

We measured general stress, indicating everyday hassles and concern about the future, with the Standard Stress Scale (SSS; Gross and Seebaß 2014; Sample item: "I often think about problems"). Using a 5-point-Likert-type scale (from $1-$ I fully disagree to $5-$ I fully agree), the scale was sufficiently reliable before $(\alpha=0.66)$ and after $(\alpha=0.71)$ the manipulation.

\subsubsection{Subjective vitality}

A feeling of energy and liveliness was measured with the Subjective Vitality Scale (SVS; Ryan and Frederick 1997; German version by Bertrams et al. 2019; Sample item: "I feel energized right now"). It consists of six items measured on a 7-point-Likert-type scale (from 1-I fully disagree to 7 - I fully agree; $\alpha_{t 1}=0.81, \alpha_{t 2}=0.85$ ).

\subsubsection{Restoration outcome}

We measured restoration as the subjectively experienced reduction of stress with the restoration outcome scale (Korpela et al. 2008; Sample item: "My concentration and alertness clearly increased"). Using a 5-point-Likert-type scale (from 1 -I fully disagree to 5-I fully agree); the scale was reliable $(\alpha=0.82)$.

\subsubsection{Perceived restorativeness}

We measured perceived restorativeness of the forest scenery with the Perceived Restorativeness Scale (PRS-11; Pasini et al. 2014; Sample item: "This place is fascinating"), which is based on ART (Kaplan et al. 1989). It is assessed with eleven items on a 5-point-Likert-type scale (from 1-I fully disagree to $5-$ I fully agree; $\alpha=0.77$ ).

\subsubsection{Demographic information}

We asked participants to indicate their gender, age, formal education, and study major. We also asked them to indicate whether they grew up and lived in rather rural or urban settings. In addition, we asked them for previous experience in VR (yes vs. no) as well as currently experienced motion sickness (yes vs. no).

\subsection{Sample and procedure}

Fifty-two participants (32 female, 19 male, 1 diverse, $M_{\text {age }}=24.2$ years, $\mathrm{SD}_{\text {age }}=3.7$ ) were recruited via messenger services and social network sites as well as on-campus of the research facility. Two participants were excluded: One participant did not want her data to be used for scientific purposes, the other encountered technical difficulties during the VR experience. In the VR group, thirteen participants indicated that they had previous experience with VR, and thirteen participants reported feelings of motion sickness. We provide analyses of previous experience and motion in the supplemental material.

The study was advertised as a study including "a walk through urban nature" and participants signed up to meet at a specific date and place. They were randomly assigned to either the physical forest condition or the virtual forest condition, resulting in twenty-five participants in each condition. Participants met the experimenter on campus and learned that they were "going for a short nature walk through adjacent forest" or that they were going to the VR laboratory for a "short Virtual Reality nature walk". They were explicitly told that they could withdraw at this point if they desired, but all participants remained in the study. Before either intervention started, participants were briefed, signed a consent form, and filled in a questionnaire ( $t 1)$ that included measures of affect, subjective vitality, and stress. Subsequently, the forest intervention was conducted.

\subsubsection{Physical condition}

Participants in the physical condition were brought to the starting point of the walk, located at the junction between the urban forest and the university's campus, where they received instructions about which way to walk. Thus, each person walked the way on their own. The circular path was easy to follow, and participants were prompted to take as much time as they needed. On average, participants returned to the start of the walk after $M=5.7 \mathrm{~min}(\mathrm{SD}=1.38$; $\operatorname{Min}=3 \mathrm{~min}, \operatorname{Max}=9.5 \mathrm{~min}$ ). Then from there, they returned to campus with the experimenter and filled in the second part of the questionnaire $(t 2)$.

The physical urban forest was a small patch of forest directly next to the university's campus, connecting the campus with an adjacent hospital and residential area. Within walking distance, people reached a scenery characterized by different trees, shrubs, and green plants, criss-crossed by various non-paved foot-paths. During summer, the paths are largely covered by the canopies, and built environment is only sporadically visible during the walk (e.g. a wooden hut or the walls of a former fort).

\subsubsection{VR condition}

Participants in the VR condition were brought to the VR laboratory, which is located in the basement of a building on campus, providing some physical movement among the VR group participants. Before they started the VR intervention, the experimenter instructed on how to use the controllers. They then received instructions about which way to go 
(including landmarks) so that they could walk the way on their own. The circular path in the VR was easy to follow (no participant got lost), and participants were prompted to take as much time as they needed. On average, participants returned to the start after $M=6.93 \mathrm{~min}(\mathrm{SD}=1.11$; $\operatorname{Min}=5.5 \mathrm{~min}, \operatorname{Max}=9.5 \mathrm{~min}$ ).

The VR environment was realized in the departments' VR laboratory, which is located in a quiet and visually shielded room with artificial light in the basement of an office building. At a given time, only the participant and the experimenter were present in the room. This laboratory consists of a high-performance PC with a Zotac GeForce GTX 1080 graphics card (8 GB RAM) and an Intel i7-7700 K main processor with $4 \mathrm{GHz}$ and $16 \mathrm{~GB}$ RAM, running on Windows 10. The VR unit used was an OculusRift head-mounted display with its two-hand controlling device and the respective sensors. Participants were asked to remain seated on an office chair, but they could move their body, arms, and head at their own discretion.

The VR environment was programmed by the experimenters, based on the properties to the physical environment, using the editor function of "The Elder Scrolls V: Skyrim Special Edition" (Bethesda Softworks LLC 2019). As the physical environment, the VR environment was a forest scenery including paths, a wooden hut, and stairs. As can be seen in Fig. 1 and in the supplemental material D, we realized a comparable setting with similar density of plants, elevation of tracks, and weather conditions (i.e. relatively sunny with few clouds for all participants). To increase comparability, bird sounds were played in the VR environment, and participants heard footstep sounds while moving. To increase comparability regarding physical exercise, participants walked a couple of minutes to the laboratory and back to the point where they completed the second part of the questionnaire.

The study was conducted in line with the ethical standards of the DGPs (German Psychological Society) and local ethics standards. There was no deception involved, participants were fully informed before the start of the study (including the information about data policies, motion sickness, and the right to withdraw at any time), and debriefed after the study. There were no measures or operations involved that likely affected emotional experience beyond usual everyday changes in affect.

\section{Results}

\subsection{Descriptive statistics}

Means $(M)$ and standard deviations (SD) of the main study variables are displayed in Table 1. Effects of the experimental condition are displayed in Figs. 2 and 3.

\subsubsection{Effects of physical and virtual nature experience on positive and negative affect}

We first tested whether participants' positive and negative affective states changed after either forest walk. Using a $2 \times 2$ repeated-measures ANOVA with time as a

Table 1 Descriptive statistics of the outcome variables

\begin{tabular}{llllll}
\hline Measure & \multicolumn{2}{l}{ Physical } & & \multicolumn{2}{l}{ VR } \\
& $M$ & SD & & $M$ & SD \\
\hline Nature environment & & & & \\
Positive affect $t 1$ & 3.34 & 0.76 & & 3.19 & 0.64 \\
Positive affect $t 2$ & 3.58 & 0.85 & & 3.37 & 0.72 \\
Negative affect $t 1$ & 1.45 & 0.45 & & 1.56 & 0.76 \\
Negative affect $t 2$ & 1.18 & 0.25 & & 1.34 & 0.60 \\
Stress $t$ 1 & 2.48 & 0.41 & & 2.60 & 0.49 \\
Stress $t 2$ & 2.43 & 0.35 & & 2.55 & 0.56 \\
Subjective vitality $t 1$ & 4.99 & 1.07 & & 4.81 & 1.09 \\
Subjective vitality $t 2$ & 5.49 & 1.03 & & 4.79 & 1.22 \\
Restoration outcome & 3.73 & 0.67 & & 3.35 & 0.92 \\
Perceived restorativeness & 3.50 & 0.69 & & 3.34 & 0.61 \\
\hline
\end{tabular}

$N=50$
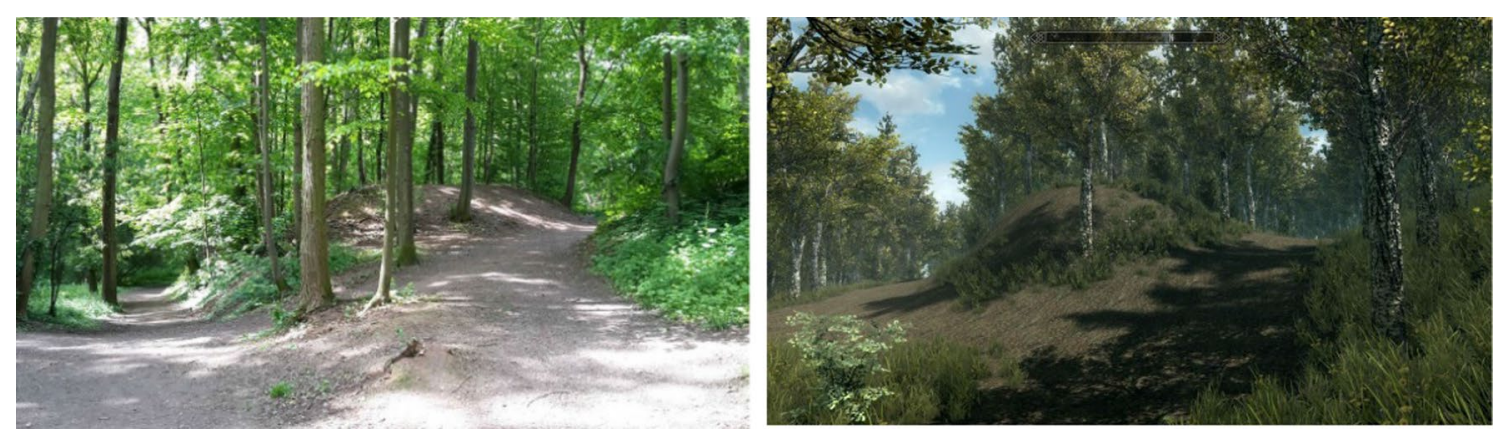

Fig. 1 The physical forest (left panel) and the VR forest (right panel). Additional screenshots are summarized in the online supplemental material 
Fig. 2 Effects of the environment and time on the respective outcome variables. Error bars represent the standard deviations

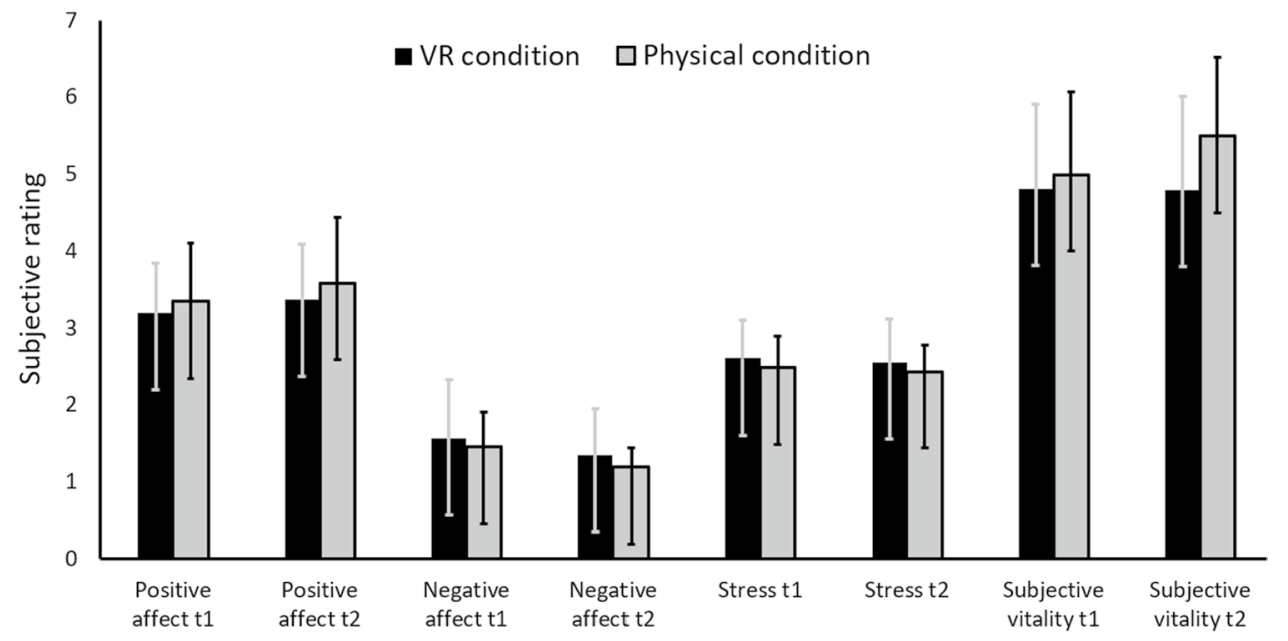

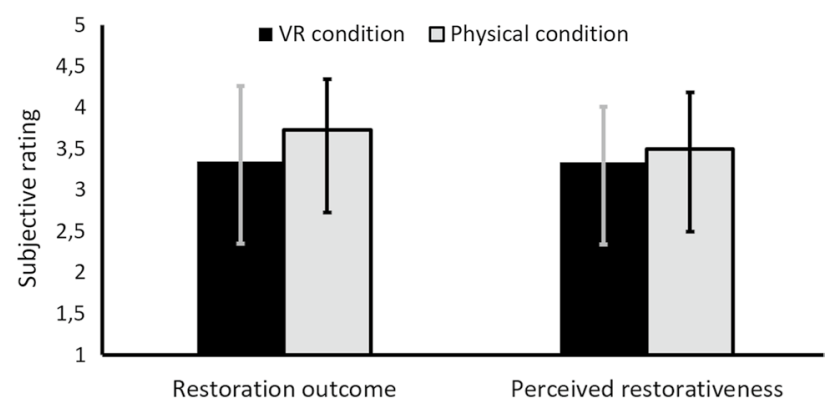

Fig. 3 Effects of the experimental condition on restoration outcome and perceived restorativeness. Error bars represent standard deviations

within-subjects factor and environment (physical vs. VR) as a between-subjects factor revealed a main effect of time, $F(1,48)=7.16, p=0.01, \eta_{p}^{2}=0.13$. Across both environments, participants reported being more positive after the nature intervention $(M=3.47, \mathrm{SD}=0.78)$ than before $(M=3.27, \mathrm{SD}=0.70)$. Effect sizes (from $t 1-t 2)$ were medium-sized within the physical (Cohen's $d=0.49$ ) and small- to medium-sized within the VR conditions (Cohen's $d=0.30$ ), but they were not significantly different as there was neither a main effect of environment, $F<1$, nor an interaction between time and environment, $F<1$.

With regard to negative affect, there was a main effect of time, $F(1,48)=24.01, p<0.001, \eta_{p}{ }^{2}=0.33$. Across both environments, participants reported less negative affect after the nature intervention $(M=1.26, \mathrm{SD}=0.46)$ than before $(M=1.51, \mathrm{SD}=0.62)$. Effect sizes (from $t 1-t 2$ ) were large within the physical (Cohen's $d=0.81$ ) and medium to large within the VR conditions (Cohen's $d=0.59$ ), but they were not significantly different as there was neither a main effect of environment, $F<1$, nor an interaction between time and environment, $F<1$.

\subsubsection{Effects of physical and virtual nature experience on subjective vitality}

Using a similar $2 \times 2$ repeated-measures ANOVA with time as within-subjects factor and environment (physical vs. VR) as between-subjects factor revealed a non-significant effect of time, $F(1,48)=2.99, p=0.09, \eta_{p}^{2}=0.06$. As the effect size suggests, however, across both environments, participants reported slightly higher subjective vitality after the nature intervention $(M=5.14, \mathrm{SD}=1.17)$ than before $(M=4.89, \mathrm{SD}=1.07)$. There was also no main effect of environment, $F(1,48)=2.46, p=0.12, \eta_{p}^{2}=0.05$. The interaction of time and environment was non-significant, $F(1,48)=3.33, p=0.07, \eta_{p}^{2}=0.07$. However, only within the physical condition, there was an effect on subjective vitality, $F(1,48)=6.31, p=0.015, \eta_{p}^{2}=0.12$, but not in the VR condition, $F<1, \eta_{p}^{2}=0.00$.

\subsubsection{Effects of physical and virtual nature experience on stress}

Using a repeated-measures ANOVA with time as the within-subjects factor and environment (physical vs. VR) as a between-subjects factor revealed a non-significant effect of time, $F(1,48)=3.7, p=0.06, \eta_{p}^{2}=0.07$. Across both environments, participants reported slightly lower subjective stress after the nature intervention $(M=2.49$, $\mathrm{SD}=0.46)$ than before $(M=2.54, \mathrm{SD}=0.45)$. Effect sizes (from $t 1-t 2$ ) were small-to-medium-sized both within the physical (Cohen's $d=0.27$ ) and the VR conditions (Cohen's $d=0.27$ ), but they were not significantly different as there was neither a main effect of environment, $F<1$, nor an interaction between time and environment, $F<1$. 


\subsubsection{Effects of physical and virtual nature experience on restoration}

To test the difference between VR and physical nature walk with regard to restoration, we submitted the ROS to a t-test for independent samples. There was no significant effect between the groups, $t(48)=1.7, p=0.10, d=0.48$. The medium-sized effect suggests, however, that participants felt slightly more restored after the physical walk $(M=3.73$, $\mathrm{SD}=0.67)$ than after the VR intervention $(M=3.35$, $\mathrm{SD}=0.92)$.

\subsubsection{Effects of physical and virtual nature experience on perceived restorativeness}

To test the difference between VR and physical environments with regard to perceived restorativeness, we submitted the PRS to a t-test for independent samples. The test revealed that both environments were perceived as similarly restorative $\left(M_{\text {physical }}=3.50, \mathrm{SD}=0.69 ; M_{\mathrm{VR}}=3.34\right.$, $\mathrm{SD}=0.61), t<1$.

\subsection{Explorative analysis}

\subsubsection{Effects of motion sickness and previous VR experience}

In order to test whether motion sickness and previous VR experience affected the effectiveness of the VR intervention, we ran a MANOVA with motion sickness (yes vs. no) and VR experience (yes vs. no) as independent variables. We used difference scores $\left(M_{\text {diff }}=t 1-t 2\right)$ of positive affect, negative affect, stress, and subjective vitality as well as the scores of restoration outcome and perceived restorativeness as dependent variables. We used MANOVA (instead of ANOVA as above) to economically explore potential effects of motion sickness and previous VR experience.

Results revealed an overall effect of motion sickness, $F(6,16)=3.59, p=0.019, \eta_{p}^{2}=0.57$. Positive affect was slightly more increased after the VR intervention when participants experienced no motion sickness $(M=-0.42$, $\mathrm{SD}=0.56$ ), compared to those reporting motion sickness $(M=0.04, \mathrm{SD}=0.58), p=0.07, \eta_{p}^{2}=0.15$. Similarly, we found a tendency for lower restoration when participants indicated motion sickness $(M=2.97, \mathrm{SD}=0.96)$ compared with no motion sickness $(M=3.75, \mathrm{SD}=0.71), p=0.053$, $\eta_{p}^{2}=0.17$. Also, participants who indicated motion sickness perceived the VR environment as less restorative $(M=3.10$, $\mathrm{SD}=0.62)$ than those not indicating motion sickness $(M=3.61, \mathrm{SD}=0.50), p=0.03, \eta_{p}^{2}=0.20$. There were no main effects of motion sickness on negative affect, subjective stress, and subjective vitality, all $F \mathrm{~s}<1$. There were also no main effects of previous VR experience on any of the dependent variables, all $F$ s $<1$.

An explorative analysis, which can be found in the supplemental material $\mathrm{B}$, addresses the role of motion sickness and previous experience in VR for the assessed outcomes. Supplemental material $\mathrm{C}$ provides the main data analysis with exclusion of participants reporting motion sickness.

\section{Discussion}

The reported study suggests that across conditions, forest experiences increased positive and decreased negative affect. Also, perceived subjective vitality increased slightly and perceived daily stress decreased slightly, corroborating to H1. The latter effects, though, were not significant, but their effect sizes were small to medium. Thus, both short-term interventions had favourable effects on reported well-being measures, but their strength is subject for future research. For example, future studies could use measures of stress that assess phasic (i.e. situationally variant) rather than tonic (i.e. perceived chronic day-to-day stress, as the measure we used taps into). We used the subjective stress scale because the subjective experience of chronic stress is especially relevant for finding coping strategies. Also, previous research suggested that perceiving one's daily stress can also change due to a VR intervention (Reese et al. 2021). For between subject variations, however, a more sensitive measure may be useful.

The comparison of the urban forest walk and the VR walk yielded no significant differences in restorative outcomes and perceived restorativeness although participants in the physical condition indicated a slightly stronger restoration, as indicated by a (albeit non-significant) medium-sized effect. Also, effects were slightly larger within the physical compared to the VR condition, as indicated by larger Cohen's $d$ s. However, as the interactions were non-significant, we assume these differences in effect size of minor relevance, and therefore, they lend only partial support to $\mathrm{H} 2$ and $\mathrm{H} 3$.

So, is "digital shinrin-yoku" a useful application for wellbeing? Our data indicate that-when the characteristics of environments are highly similar-a nature walk through a virtual forest evokes roughly similar beneficial outcomes than a walk through a real forest. Resonating with previous studies (Chirico and Gaggioli 2019; Mattila et al. 2020), this study suggests that VR nature experiences may be an appropriate compensation for people who cannot visit real nature for whatever reason. The analysis in supplement $\mathrm{C}$, in which we report findings excluding people with motion sickness, supports this notion even more strongly, but should be interpreted with caution given the small and unequal sample size. 
Although we believe that our urban forest and the virtual environment were very similar in terms of visual appearance and content, there are two major limitations of the current study. First and foremost, participants in in the physical forest condition were walking while participants in the VR condition were seated. However, both could move through the forest at their own pace. A feeling of walking-possibly on soft ground-may have even increased the restoring effect (cf. Calogiuri et al. 2018) so that the similarity of effects between the two conditions is even more remarkable. Saying that, there is to our knowledge little research directly comparing participants who are seated in a nature setting with those who are walking (but see, for example, Mygind et al. 2019, for meta-analytic differences in stress response for studies with seated or walking nature interventions). Walking is generally associated with therapeutic effects (Crone 2007) and people generally see walking as something positive (Rybråten et al. 2019). Only few studies have looked at specific effects of VR walking on well-being outcomes (Barton and Pretty 2010), suggesting that even light doses of outdoor exercise (e.g. 5-min walking) can result in increased self-esteem and mood. In an unpublished qualitative study from our laboratory, we asked participants to either walk through a physical forest or look for a place to sit down in the forest (Authors unpublished manuscript). Both groups of forest bathers reported heightened nature connectedness and relaxation. Future operationalizations could compare participants navigating through VR either seated or walking on a treadmill. Although the difference in seating and walking in our conditions is unfortunate for a direct comparison, it emphasizes the effectiveness for VR nature interventions as well-being outcomes were similar despite lower physical activity in VR. This strongly suggests beneficial effects of virtual shinrin-yoku for immobile and/or older people as well (see also Reese et al. 2021; Kabisch et al. 2017; Mygind et al. 2019).

Second, we suggest that future research more closely inspects the role of motion sickness (e.g. Dziuda et al. 2014; Kim et al. 2018) with regard to restoration effects. We have run exploratory analyses testing the effects of motion sickness (and previous VR experience) on our well-being indicators. Although caution is needed due to its exploratory character, we found that, indeed, people experiencing motion sickness perceived the VR environment as significantly less restorative, reported significantly lower restoration, and weaker change in positive affect compared to those indicating no motion sickness. With regard to subjective vitality, stress and negative affect, however, there were no differences in change between those reporting motion sickness and those who did not. Again, these findings should be treated with much caution, given the relatively small sample. Yet, even though half of our VR participants reported motion sickness, the similar outcomes of our two interventions again emphasizes the beneficial effects of the VR intervention. If participants and/or VR developers handle motion sickness in future applications, VR nature experiences may become even more restorative than today - as also indicated by the analysis in supplement C. VR applications could then represent innovative tools for promoting well-being. In terms of digitalizing shinrin-yoku, future research should continue testing the influences of the five senses that contribute most to the therapeutic effects of forest bathing (cf. Hedblom et al. 2019).

Another issue we believe deserves critical reflection is related to the sample size — both with regard to the current study, but also to VR research in general. Very often, VR research utilizes samples that are rather small (e.g. with $\mathrm{Ns}$ ranging from 18 to 50 in Anderson et al. 2017; Calogiuri et al. 2018; Chirico and Gaggioli 2019; Hu et al. 2021; Chan et al. in press) in comparison to, for example, online surveys. Many studies-including ours-may thus lack statistical power and thus result in questionable validity. For example, the achieved statistical power (post-hoc) of our statistical tests ranged from $1-\beta=0.51$ for the $\mathrm{t}$-test comparing perceived restoration after physical vs. VR walk, to $1-\beta>0.95$ for the post-hoc tests of the repeated measure analyses. As a consequence, between-subjects effects (and a lack thereof) obtained here, and probably in other studies, are subject to caution, and should be substantiated by future studies. Saying that, however, we also think that feasibility needs to be considered. VR studies (and other resource-intense experiments) require much more time and (wo)man power than survey studies so that accumulative evidence of various weakly-powered VR studies from different laboratories can be helpful and might provide at least some confidence. However, as has been discussed in other resource-intense research fields (e.g. fMRI studies; Yarkoni 2009), the best way to increase power is to obtain larger samples.

Finally, we identified two research fields that could more strongly benefit from VR nature representations. First, we think that more research on the VR effects on health could apply (neuro-)physiological measures to test effects on objective health outcomes. There is emerging research doing so (e.g. with regard to brain activity measures and heart rate; e.g. Hu et al. 2021; Chan et al. in press), and we believe that the usability of VR in health settings could benefit from further tests of VR versus physical settings. When such in-depth investigations confirm benefits, results may translate into design concepts for digital health interventions. For example, with more and more people having access to such digital technologies, digital short-term interventions may reduce intake of medication that could have unwanted side effects. Second, future research should more strongly investigate the role of VR representations on pro-environmental behaviour. For example, recent evidence suggests that presenting information on global environmental change of place can elicit 
emotional responses (Wullenkord et al. 2020), and emotions are strong predictors of pro-environmental action (e.g. Harth et al. 2013; Landmann and Rohmann 2020; Reese and Jacob 2015). Using VR technology may proof useful to visualize and allow immersion into change, for example with regard to displaying resource use (Chirico et al. 2021). Similarly, we believe that exposure to virtual nature and underscoring that nature is important for well-being may foster the belief that nature needs protection. Relatedly, (real and virtual) nature experiences can increase connectedness to nature, which is robustly related to both well-being and pro-environmental (for meta-analyses, see, for example, Mackay and Schmitt 2019; Pritchard et al. 2019).

\section{Conclusion}

Taken together, the current study suggests that a short virtual forest walk can increase well-being similarly to a walk in a comparable physical forest. It may therefore be a useful substitute if necessary or favoured. However, we are (yet) convinced that substituting should not be the norm-as there are various health-related reasons to go for a real walk in physical nature. But when time is scarce or mobility is limited, a VR nature experience may be better than none.

Supplementary Information The online version contains supplementary material available at https://doi.org/10.1007/s10055-022-00631-9.

Acknowledgements This research did not receive any specific grant from funding agencies in the public, commercial, or not-for-profit sectors.

Author contributions Blinded for review.

Funding Open Access funding enabled and organized by Projekt DEAL.

\section{Declarations}

Conflict of interest The authors declare that they have no conflict of interest.

Ethical standards The study was conducted in line with the ethical standards of the DGPs (German Psychological Society) and local ethics standards. There was no deception involved, participants were fully informed before the start of the study (including the information about data policies, motion sickness, and the right to withdraw at any time), and debriefed after the study. There were no measures or operations involved that likely affected emotional experience beyond usual everyday changes in affect.

Open Access This article is licensed under a Creative Commons Attribution 4.0 International License, which permits use, sharing, adaptation, distribution and reproduction in any medium or format, as long as you give appropriate credit to the original author(s) and the source, provide a link to the Creative Commons licence, and indicate if changes were made. The images or other third party material in this article are included in the article's Creative Commons licence, unless indicated otherwise in a credit line to the material. If material is not included in the article's Creative Commons licence and your intended use is not permitted by statutory regulation or exceeds the permitted use, you will need to obtain permission directly from the copyright holder. To view a copy of this licence, visit http://creativecommons.org/licenses/by/4.0/.

\section{References}

Alvarsson JJ, Wiens S, Nilsson ME (2010) Stress recovery during exposure to nature sound and environmental noise. Int J Environ Res Public Health 7(3):1036-1046. https://doi.org/10.3390/ijerp h7031036

Anderson AP, Mayer MD, Fellows AM, Cowan DR, Hegel MT, Buckey JC (2017) Relaxation with immersive natural scenes presented using virtual reality. Aerosp Med Human Perform 88(6):520-526. https://doi.org/10.3357/AMHP.4747.2017

Authors. The experience of connectedness with nature while sitting and walking. Unpublished manuscript

Barton J, Pretty J (2010) What is the best dose of nature and green exercise for improving mental health? A multi-study analysis. Environ Sci Technol 44(10):3947-3955

Becker DA, Browning MHEM, Kuo M, Van Den Eeden SK (2019) Is green land cover associated with less health care spending? Promising findings from county-level Medicare spending in the continental United States. Urb For Urb Green. https://doi.org/10. 1016/j.ufug.2019.02.012

Berman MG, Jonides J, Kaplan S (2008) The cognitive benefits of interacting with nature. Psychol Sci 19(12):1207-1212. https:// doi.org/10.1111/j.1467-9280.2008.02225.x

Berto R (2005) Exposure to restorative environments helps restore attentional capacity. J Environ Psychol 25(3):249-259. https:// doi.org/10.1016/j.jenvp.2005.07.001

Bertrams A, Dyllick-Brenzinger T, Englert C, Krispenz A (2019) German adaptation of the subjective vitality scales (SVS-G), accepted version. Open Psychol. https://doi.org/10.31234/osf.io/c3bwu

Bethesda Softworks LLC (2019) Maryland, US

Bratman GN, Anderson CB, Berman MG, Cochran B, de Vries S, Flanders J, Folke C, Frumkin H, Gross JJ, Hartig T, Kahn PH, Kuo M, Lawler JJ, Levin PS, Lindahl T, Meyer-Lindenberg A, Mitchell R, Ouyang Z, Roe J, Scarlett L, Smith JR, van den Bosch M, Wheeler BW, White MP, Zheng H, Daily GC (2019) Nature and mental health: an ecosystem service perspective. Sci Adv 5(7):0903. https://doi.org/10.1126/sciadv.aax0903

Breyer B, Bluemke M (2016) Deutsche version der positive and negative affect schedule PANAS (GESIS panel)

Brivio E, Serino S, Cousa EN, Zini A, Riva G, De Leo G (2021) Virtual reality and 360 panorama technology: a media comparison to study changes in sense of presence, anxiety, and positive emotions. Virtual Real 25(2):303-311

Brown DK, Barton JL, Gladwell VF (2013) Viewing nature scenes positively affects recovery of autonomic function following acutemental stress. Environ Sci Technol 47(11):5562-5569. https://doi. org/10.1021/es305019p

Browning MH, Mimnaugh KJ, van Riper CJ, Laurent HK, LaValle SM (2019) Can simulated nature support mental health? Comparing short, single-doses of 360-degree nature videos in virtual reality with the outdoors. Front Psychol. https://doi.org/10.3389/fpsyg. 2019.02667

Calogiuri G, Litleskare S, Fagerheim KA, Rydgren TL, Brambilla E, Thurston M (2018) Experiencing nature through immersive virtual environments: environmental perceptions, physical 
engagement, and affective responses during a simulated nature walk. Front Psychol 8:2321

Chan SHM, Qiu L, Esposito G, Mai KP, Tam KP, Cui J (in press) Nature in virtual reality improves mood and reduces stress: evidence from young adults and senior citizens. Virtual Real

Chirico A, Gaggioli A (2019) When virtual feels real: comparing emotional responses and presence in virtual and natural environments. Cyberpsychol Behav Soc Netw 22(3):220-226

Chirico A, Scurati GW, Maffi C, Huang S, Graziosi S, Ferrise F, Gaggioli A (2021) Designing virtual environments for attitudes and behavioral change in plastic consumption: a comparison between concrete and numerical information. Virtual Real 25(1):107-121

Crone D (2007) Walking back to health: a qualitative investigation into service users' experiences of a walking project. Issues Ment Health Nurs 28(2):167-183. https://doi.org/10.1080/01612 840601096453

De Lange AH, Taris TW, Kompier MA, Houtman IL, Bongers PM (2003) "The very best of the millennium": longitudinal research and the demand-control-(support) model. J Occup Health Psychol 8(4):282-305

Dziuda Ł, Biernacki MP, Baran PM, Truszczyński OE (2014) The effects of simulated fog and motion on simulator sickness in a driving simulator and the duration of after-effects. Appl Ergon 45(3):406-412

Engemann K, Pedersen CB, Arge L, Tsirogiannis C, Mortensen PB, Svenning J-C (2019) Residential green space in childhood is associated with lower risk of psychiatric disorders from adolescence into adulthood. Proc Natl Acad Sci. https://doi.org/10. 1073/pnas. 1807504116

Franco LS, Shanahan DF, Fuller RA (2017) A review of the benefits of nature experiences: more than meets the eye. Int J Environ Res Public Health 14(8):864. https://doi.org/10.3390/ijerph1408 0864

Gamble KR, Howard JH Jr, Howard DV (2014) Not just scenery: viewing nature pictures improves executive attention in older adults. Exp Aging Res 40(5):513-530. https://doi.org/10.1080/03610 73X.2014.956618

Gerber SM, Jeitziner MM, Wyss P, Chesham A, Urwyler P, Müri RM, Jakob SM, Nef T (2017) Visuo-acoustic stimulation that helps you to relax: a virtual reality setup for patients in the intensive care unit. Sci Rep 7(1):1-10. https://doi.org/10.1038/ s41598-017-13153-1

Gladwell VF, Brown DK, Barton JL, Tarvainen MP, Kuoppa P, Pretty J, Suddaby JM, Sandercock GRH (2012) The effects of views of nature on autonomic control. Eur J Appl Physiol 112(9):33793386. https://doi.org/10.1007/s00421-012-2318-8

Gross C, Seebaß K (2014). The standard stress scale (SSS): measuring stress in the life course. In: H-P Blossfeld, J v Maurice, M Bayer, J Skopek (Eds.). Methodological issues of longitudinal surveys. Springer VS, Wiesbaden, Germany, pp 233-249

Hansen MM, Jones R, Tocchini K (2017) Shinrin-yoku (forest bathing) and nature therapy: a state-of-the-art review. Int J Environ Res Public Health 14(8):851

Harth NS, Leach CW, Kessler T (2013) Guilt, anger, and pride about in-group environmental behaviour: different emotions predict distinct intentions. J Environ Psychol 34:18-26

Hartig T, Böök A, Garvill J, Olsson T, Gärling T (1996) Environmental influences on psychological restoration. Scand J Psychol 37(4):378-393. https://doi.org/10.1111/j.1467-9450.1996.tb006 70.x

Hartig T, Mitchell R, de Vries S, Frumkin H (2014) Nature and health. Annu Rev Public Health 35(1):207-228. https://doi.org/10.1146/ annurev-publhealth-032013-182443

Hauru K, Lehvävirta S, Korpela K, Kotze DJ (2012) Closure of view to the urban matrix has positive effects on perceived restorativeness in urban forests in Helsinki, Finland. Landsc Urb Plan 107(4):361-369

Hedblom M, Gunnarsson B, Iravani B, Knez I, Schaefer M, Thorsson P, Lundström JN (2019) Reduction of physiological stress by urban green space in a multisensory virtual experiment. Sci Rep. https:// doi.org/10.1038/s41598-019-46099-7

Hu M, Simon M, Fix S, Vivino AA, Bernat E (2021) Exploring a sustainable building's impact on occupant mental health and cognitive function in a virtual environment. Sci Rep 11(1):1-13

Ilies R, Schwind KM, Wagner DT, Johnson MD, DeRue DS, Ilgen DR (2007) When can employees have a family life? The effects of daily workload and affect on work-family conflict and social behaviors at home. J Appl Psychol 92(5):1368-1379

Kabisch N, van den Bosch M, Lafortezza R (2017) The health benefits of nature-based solutions to urbanization challenges for children and the elderly: a systematic review. Environ Res 159:362-373. https://doi.org/10.1016/j.envres.2017.08.004

Kaplan R, Kaplan S, Brown T (1989) Environmental preference: a comparison of four domains of predictors. Environ Behav 21(5):509-530

Kaplan S (1995) The restorative benefits of nature: toward an integrative framework. J Environ Psychol 15(3):169-182. https:// doi.org/10.1016/0272-4944(95)90001-2

Kim HK, Park J, Choi Y, Choe M (2018) Virtual reality sickness questionnaire (VRSQ): motion sickness measurement index in a virtual reality environment. Appl Ergon 69:66-73

Korpela K, Ylén M, Tyrväinen L, Silvennoinen H (2008) Determinants of restorative experiences in everyday favourite places. Health Place 14:636-652

Kuo M (2015) How might contact with nature promote human health? Promising mechanisms and a possible central pathway. Front Psychol. https://doi.org/10.3389/fpsyg.2015.01093

Kuo FE, Sullivan WC (2001) Aggression and violence in the inner city: effects of environment via mental fatigue. Environ Behav 33(4):543-571. https://doi.org/10.1177/00139160121973124

Landmann H, Rohmann A (2020) Being moved by protest: collective efficacy beliefs and injustice appraisals enhance collective action intentions for forest protection via positive and negative emotions. J Environ Psychol 71:101491

Mackay CML, Schmitt MT (2019) Do people who feel connected to nature do more to protect it? A meta-analysis. J Environ Psychol. https://doi.org/10.1016/j.jenvp.2019.101323

Mattila O, Korhonen A, Pöyry E, Hauru K, Holopainen J, Parvinen P (2020) Restoration in a virtual reality forest environment. Comput Hum Behav 107:106295. https://doi.org/10.1016/j.chb. 2020.106295

Menardo E, Brondino M, Hall R, Pasini M (2019) Restorativeness in natural and urban environments: a meta-analysis. Psychol Rep. https://doi.org/10.1177/0033294119884063

Mygind L, Kjeldsted E, Hartmeyer RD, Mygind E, Bølling M, Bentsen $\mathrm{P}$ (2019) Immersive nature-experiences as health promotion interventions for healthy, vulnerable, and sick populations? A systematic review and appraisal of controlled studies. Front Psychol. https://doi.org/10.3389/fpsyg.2019.00943

Ohly H, White MP, Wheeler BW, Bethel A, Ukoumunne OC, Nikolaou V, Garside R (2016) Attention restoration theory: a systematic review of the attention restoration potential of exposure to natural environments. J Toxicol Environ Health Part B 19(7):305-343. https://doi.org/10.1080/10937404.2016.11961 55

Pasini M, Berto R, Brondino M, Hall R, Ortner C (2014) How to measure the restorative quality of environments: the PRS-11. Procedia Soc Behav Sci 159:293-297

Pritchard A, Richardson M, Sheffield D, McEwan K (2019) The relationship between nature connectedness and eudaimonic 
well-being: a meta-analysis. J Happiness Stud. https://doi.org/ 10.1007/s10902-019-00118-6

Reese G, Jacob L (2015) Principles of environmental justice and pro-environmental action: a two-step process model of moral anger and responsibility to act. Environ Sci Policy 51:88-94

Reese G, Kohler E, Menzel C (2021) Restore or get restored: the effect of control on stress reduction and restoration in virtual nature settings. Sustainability 13(4):1995

Ryan RM, Frederick C (1997) On energy, personality, and health: subjective vitality as a dynamic reflection of well-being. J Pers 65:529-565

Rybråten S, Skår M, Nordh H (2019) The phenomenon of walking: diverse and dynamic. Landsc Res 44(1):62-74

Scannell L, Gifford R (2010) Defining place attachment: a tripartite organizing framework. J Environ Psychol 30(1):1-10

Scates D, Dickinson JI, Sullivan K, Cline H, Balaraman R (2020) Using nature-inspired virtual reality as a distraction to reduce stress and pain among cancer patients. Environ Behav. https://doi.org/10. $1177 / 0013916520916259$

Stevenson MP, Schilhab T, Bentsen P (2018) Attention restoration theory II: a systematic review to clarify attention processes affected by exposure to natural environments. J Toxicol Environ Health Part B 21(4):227-268. https://doi.org/10.1080/10937404.2018. 1505571

Tanja-Dijkstra K, Pahl S, White MP, Auvray M, Stone RJ, Andrade J, May J, Mills I, Moles DR (2018) The soothing sea: a virtual coastal walk can reduce experienced and recollected pain. Environ Behav 50(6):599-625

Ulrich RS, Simons RF, Losito BD, Fiorito E, Miles MA, Zelson M (1991) Stress recovery during exposure to natural and urban environments. J Environ Psychol 11(3):201-230. https://doi.org/10. 1016/S0272-4944(05)80184-7

Valtchanov D, Ellard CG (2010) Physiological and affective responses to immersion in virtual reality: effects of nature and urban settings. J Cyberther Rehabil 3(4):359-374 van den Berg M, Maas J, Muller R, Braun A, Kaandorp W, van Lien R, van Poppel M, van Mechelen W, van den Berg A (2015) Autonomic nervous system responses to viewing green and built settings: differentiating between sympathetic and parasympathetic activity. Int J Environ Res Public Health 12(12):15860-15874. https://doi.org/10.3390/ijerph121215026

Watson D, Clark LA, Tellegen A (1988) Development and validation of brief measures of positive and negative affect: the PANAS scales. J Pers Soc Psychol 54(6):1063-1070

World Health Organization (WHO) (2019) Constitution of the World Health Organization. Retrieved from https://www.who.int/about/ who-we-are/constitution

Wullenkord MC, Heidbreder LM, Reese G (2020) Reactions to environmental changes: place attachment predicts interest in earth observation data. Front Psychol 11:1442

Yarkoni T (2009) Big correlations in little studies: inflated fMRI correlations reflect low statistical power-commentary on Vul et al. (2009). Perspect Psychol Sci 4(3):294-298

Yu CP, Lee HY, Luo XY (2018) The effect of virtual reality forest and urban environments on physiological and psychological responses. Urb For Urb Green 35:106-114. https://doi.org/10. 1016/j.ufug.2018.08.013

Zelenski JM, Dopko RL, Capaldi CA (2015) Cooperation is in our nature: nature exposure may promote cooperative and environmentally sustainable behavior. J Environ Psychol 42:24-31. https://doi.org/10.1016/j.jenvp.2015.01.005

Publisher's Note Springer Nature remains neutral with regard to jurisdictional claims in published maps and institutional affiliations. 